\title{
Implementación de la gestión del conocimiento con liderazgo y valores en una IES del Ecuador
}

\section{Implementation of knowledge management with leadership and values in an IES of Ecuador}

Teresa Angélica Altamirano Novillo. ', Paulina Elizabeth Valverde Aguirre. ${ }^{2}$, Juan Efraín Sánchez Vimos . ${ }^{3} \&$ Johanna Enith Aguilar Reyes. ${ }^{4}$

\begin{abstract}
.
DOI: https://doi.org/10.33262/cienciadigital.v3i3.2.1.780

The purpose of this research is to analyze the knowledge management capacity with leadership integrated to personal and institutional values that contribute to raising the quality of education in higher level educational centers.

Starting with the research of theoretical references on educational management, educational leadership and quality management in values; as well as discovering a critical attitude to select, process and present valuable information that each of the authors of higher education experiences, determining the roles and leadership of managers in the execution of strategic and operational plans in the investigated institution which allows us to assume with responsibility the elaboration of the institutional diagnosis in the different scenarios that shows the leadership and values as a transversal axis of the educational administration. The research helped to know more about what management, leadership and values mean, and to know the current real situation and its vision in the individual, group and institutional progress of its actors. Therefore, this institution constitutes an academic reference for current generations in ethical training and values.
\end{abstract}

Keywords: Education, leadership, values, educational management

1 Escuela Superior Politécnica de Chimborazo, Facultad de Ciencias. Riobamba, Ecuador. taltamirano@espoch.edu.ec

2 Escuela Superior Politécnica de Chimborazo, Facultad de Ciencias. Riobamba, Ecuador. Paulina.valverde@espoch.edu.ec

3 Escuela Superior Politécnica de Chimborazo, Facultad de Recursos Naturales. Riobamba, Ecuador. jusanchez@espoch.edu.ec

4 Escuela Superior Politécnica de Chimborazo, Facultad de Recursos Naturales. Riobamba, Ecuador. johannae.aguilar@espoch.edu.ec 


\section{Resumen.}

La presente investigación, tiene por objetivo analizar la capacidad de gestión del conocimiento con liderazgo integrado a los valores personales e institucionales que permitan contribuir a elevar la calidad de la educación en los centros educativos de nivel superior.

Empezando con la investigación de los referentes teóricos sobre gestión educativa, liderazgo educativo y gestión de la calidad en valores; así como descubrir una actitud crítica para seleccionar, procesar y presentar información valiosa que vivencia cada una de los autores de la educación superior, determinando los roles y liderazgo de los directivos en la ejecución de planes estratégicos y operativos en la institución investigada la cual permite asumir con responsabilidad la elaboración del diagnóstico institucional en los diferentes escenarios que se evidencia el liderazgo y valores como eje transversal de la administración educativa.

La investigación ayudó a conocer más sobre lo que significa gestión, liderazgo y valores, y conocer la situación real actual y su visión en el progreso individual, grupal e institucional de sus actores.

Por lo tanto dicha institución constituye un referente académico para las actuales generaciones en la formación ética y en valores.

Palabras claves: Educación, liderazgo, valores, gestión educativa

\section{Introducción.}

La Gestión del conocimiento es un concepto aplicado en las organizaciones, que busca transferir el conocimiento y la experiencia existente entre sus miembros, de modo que pueda ser utilizado como un recurso disponible para otros en la organización.

La Escuela de Física y Matemática pertenece a la Facultad de Ciencias de la Escuela Superior Politécnica de Chimborazo. En el último semestre se han modificado los planes analíticos, y se han hecho algunos cambios en los modelos pedagógicos, pero no se ha hecho nada en cuanto a la gestión del conocimiento.

Entonces el problema dentro de la escuela de Física y Matemática es que no nos damos cuenta del capital intelectual que se tiene dentro de ella, y a causa de esto no se lo puede difundir dentro de esta organización educativa, por lo mismo tampoco se está actuando de manera correcta en lo que es liderazgo y gestión; al valorar el capital intelectual estaremos aceptando trabajar en equipo con sólidas bases en el conocimiento de los valores y aceptando la dirección del líder. 
Esto nos beneficia ya que los alumnos podrán ver el trabajo en equipo con la práctica de todos los valores, y daremos respuesta a la encuesta en que casi todos respondieron que se enseña con el ejemplo.

Las autoridades solo se han empeñado en el proceso de autoevaluación que constituye en mejorar las ofertas académicas y también el promedio de calificaciones por cada alumno, por lo que de parte de ellas tampoco hay un apoyo en cuanto a la transferencia de conocimientos.

La ejecución del presente proyecto además de ser importante, es muy necesaria, ya que la Gestión del Conocimiento con Liderazgo y Valores de una forma más precisa es conjunto de procesos y sistemas que permiten que el Capital Intelectual de una organización en este caso de la organización educativa que es la Escuela de Física y Matemática aumente de forma significativa, mediante la gestión de sus capacidades de resolución de problemas de forma eficiente.

El aprendizaje organizativo, la Gestión del Conocimiento, el Liderazgo, los Valores y la Medición del Capital Intelectual son conceptos relacionados y complementarios. En pocas palabras, el aprendizaje organizativo es la base de una buena Gestión del Conocimiento, y la Gestión del Conocimiento es la base para la generación de Capital Intelectual y capacidades organizativas fundamentadas el Liderazgo y los Valores.

Los beneficiarios directos son los integrantes de la escuela de Física y Matemática, desde el director, los miembros de comisión de carrera, docentes de nombramiento y a contrato y los alumnos de todos los semestres.

Los beneficiarios indirectos, serán las autoridades empezando con el vicedecano y decano, luego el resto de autoridades, en fin a toda la comunidad politécnica.

Los problemas son claramente identificados, por una parte no se cuenta con personal encargado de desarrollar el capital intelectual y poder transmitirlo, la poca información acerca de lo que es la gestión del conocimiento, y luego el poco apoyo de las autoridades ante la situación.

\section{Metodología.}

\section{Método histórico.}

Este vinculado al conocimiento de las distintas etapas de los objetos en su sucesión cronológica, para conocer la evolución y desarrollo del objeto o fenómeno de investigación se hace necesario revelar su historia, las etapas principales de su desenvolvimiento y las 
conexiones históricas fundamentales.

Mediante el método histórico se analiza la trayectoria concreta de la teoría, su condicionamiento a los diferentes periodos de la historia. Los métodos lógicos se basan en el estudio histórico poniendo de manifiesto la lógica interna de desarrollo, de su teoría y allá el conocimiento más profundo de estas, de su esencia estructura lógica del objeto implica su modelación.

\section{Método deductivo.}

La deducción va de lo general a lo particular. El método deductivo es aquel que parte los datos generales aceptarlos como valederos, para deducir por medio del razonamiento lógico. Varia sus posiciones es decir parte de verdades previamente establecidas como principios generales para luego aplicar a casos individuales y comprobar así su validez.

\section{Método inductivo.}

La inducción va de lo particular a lo general. Empleamos el método inductivo cuando de la observación de los hechos particulares obtenemos proposiciones generales, o sea, es aquel que establece un principio general una vez realizado el estudio y análisis de hechos y fenómenos en particular.

La inducción es proceso mental que consiste en inferir de algunos casos particulares observados la ley general que lo rige para todos de la misma especie.

\section{Método analítico.}

Es aquel que distingue las partes de un todo y procede a la revisión ordenada de cada uno de sus elementos por separado.

Analizar significa: observar y penetrar en cada uno de las partes de un objeto que se considera como unidad. En la investigación documental es aplicable desde el principio desde el momento en que se revisa uno por uno los diversos documentos o libros que nos proporcionaran los datos buscados. El análisis es provechoso en cuanto que proporciona nuevos elementos de juicio.

\section{Método sintético.}

Consiste en reunir los diversos elementos que se analizaron anteriormente en general la síntesis y el análisis son dos fases complementarias.

La síntesis es indispensable en cuanto reúne en sus elementos y produce nuevos juicios, criterios, tesis y argumentación. 


\section{Localización y cobertura.}

Esta investigación se desarrolla y se aplicará en la Escuela de Física y Matemática de la Facultad de Ciencias perteneciente a la ESPOCH; la institución educativa está ubicada en la Panamericana Sur Km $1^{1 / 2}$ de la ciudad de Riobamba provincia de Chimborazo.

\section{Población objetivo}

Los actores involucrados en el sistema educativo, que aúnan esfuerzos y buscan aliados estratégicos para alcanzar una educación integral y de calidad, son: el director, los docentes, personal administrativo, personal de servicio y los alumnos de la Escuela de Física y Matemática de la facultad de Ciencias de la ESPOCH.

\section{Resultados.}

Después de la aplicación de encuestas a los involucrados durante el proceso, se ha podido recolectar información, y obtener los siguientes resultados:

Figura 1. El Rol del Docente.

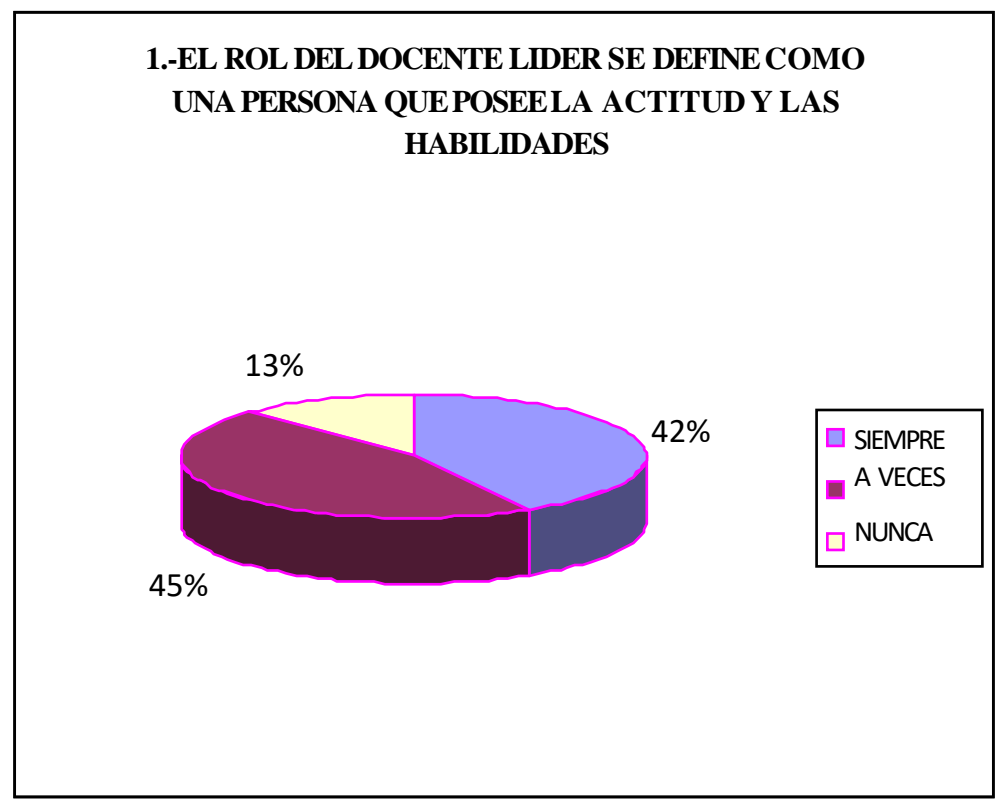

Fuente: Elaboración propia.

En la figura 1 se observa que el $45 \%$ de los profesores piensan que a veces el rol del docente líder se define como una persona que posee la actitud y las habilidades para cuestionar las órdenes existentes; mientras que el $42 \%$ piensa que siempre el rol del docente líder se define como una persona que posee la actitud y las habilidades para cuestionar las órdenes existentes; y el 13\% piensa que nunca el rol del docente líder se define como una persona que posee la actitud y las habilidades para cuestionar las órdenes existentes. 
Figura 2. La Gerencia

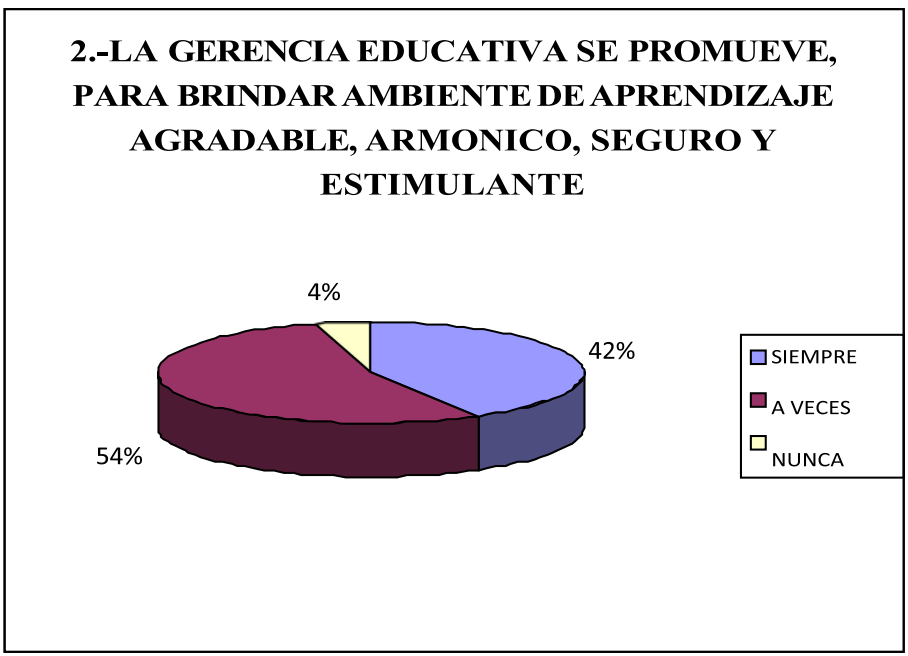

Fuente: Elaboración propia.

Con la figura 2 nos damos cuenta que el 54\% de los docentes piensa que a veces la gerencia educativa se promueve, para brindar ambiente de aprendizaje agradable, armónico, seguro y estimulante; el $42 \%$ piensa que siempre la gerencia educativa se promueve, para brindar ambiente de aprendizaje agradable, armónico, seguro y estimulante; y el restante $4 \%$ piensa que la gerencia educativa se promueve, para brindar ambiente de aprendizaje agradable, armónico, seguro y estimulante.

Figura 3. Los directivos

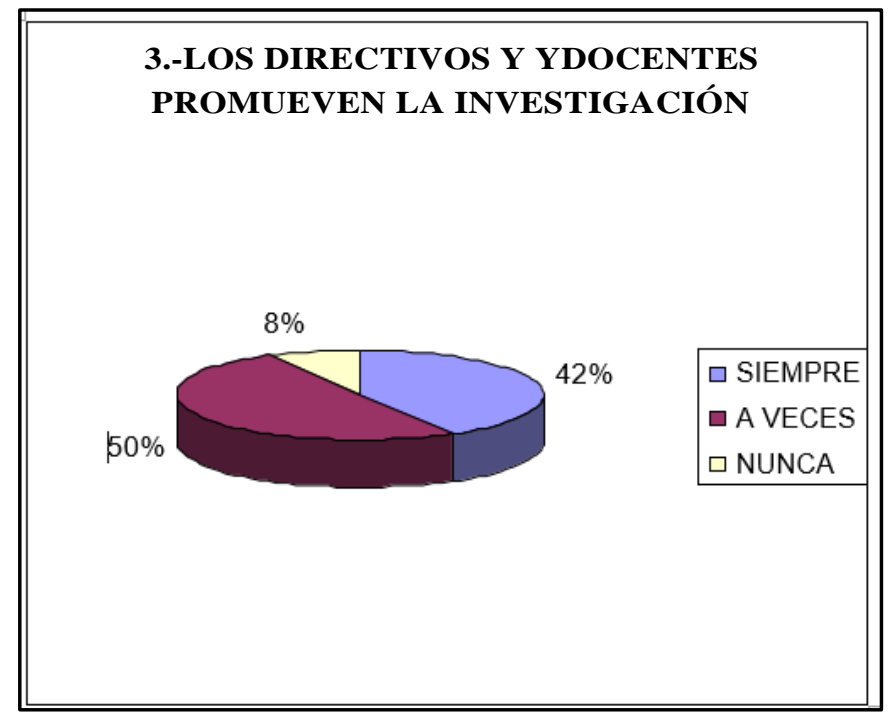

Fuente: Elaboración propia.

En la figura 3 el $50 \%$ dice que a veces los directivos y docentes promueven la 
investigación; el $42 \%$ dice que los directivos y docentes promueven la investigación; y sólo el $8 \%$ dice que nunca los directivos y docentes promueven la investigación.

Figura 4. Proceso de enseñanza

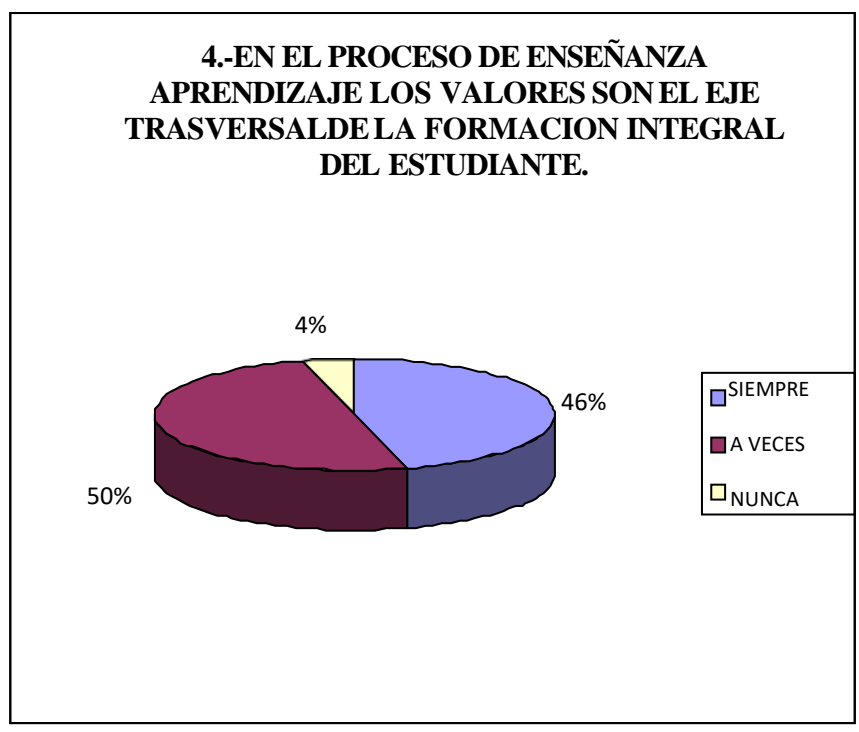

Fuente: Elaboración propia.

En la figura 4 tenemos que los docentes dicen que el $50 \%$ a veces, el $46 \%$ dice que siempre, y el $4 \%$ dice que nunca en el proceso de enseñanza-aprendizaje los valores son el eje trasversal de la formación integral del estudiante.

Figura 5.

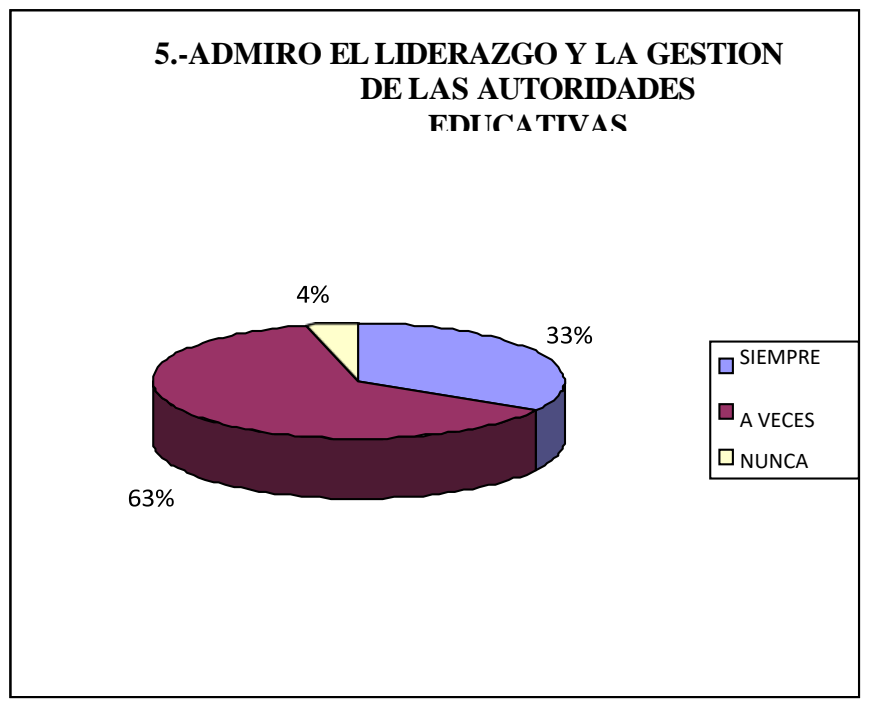

Fuente: Elaboración propia.

En la figura 5 se muestra que el $63 \%$ de docentes piensa que a veces admiran el liderazgo y 
la gestión de las autoridades educativas; el 33\% de los docentes piensa que siempre admiran el liderazgo y la gestión de las autoridades educativas; y el $4 \%$ de docentes dice que nunca admiran el liderazgo y la gestión de las autoridades educativas.

Figura 6. Los Directivos

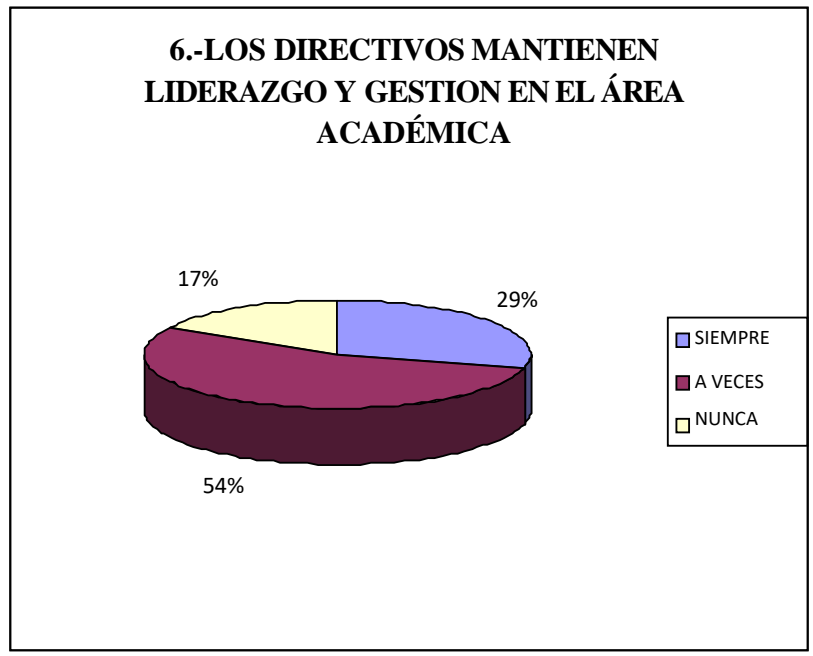

Fuente: Elaboración propia.

La figura 6 indica que el 54\% de los docentes dice que a veces los directivos mantienen liderazgo y gestión en el área académica; el 29\% de los docentes dice que siempre los directivos mantienen liderazgo y gestión en el área académica; y el 17\% de los docentes dicen que nunca los directivos mantienen liderazgo y gestión en el área académica.

Figura 7. Los Directivos

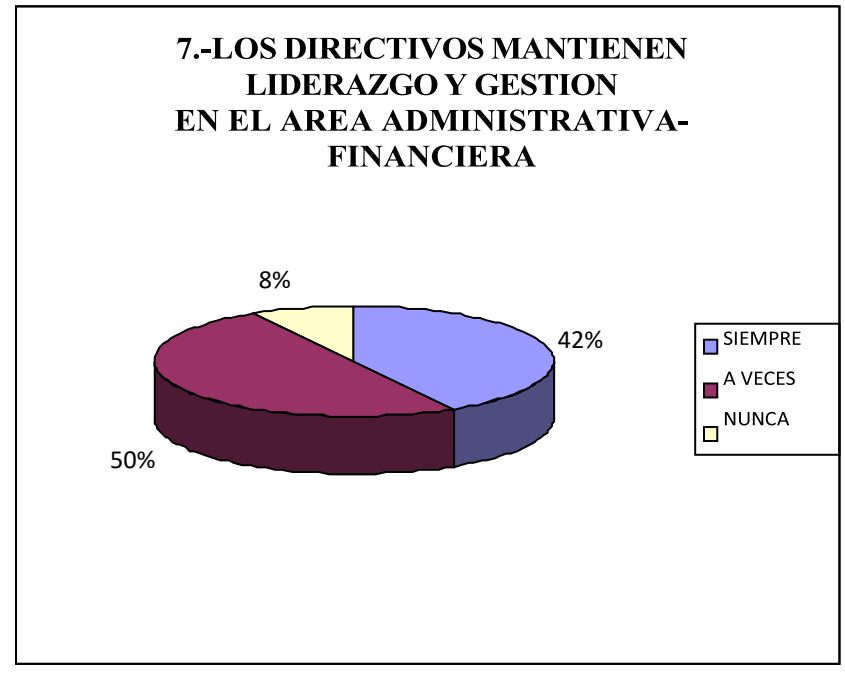

Fuente: Elaboración propia. 
La figura 7 muestra que el $50 \%$ de los docentes dicen que a veces los directivos mantienen liderazgo y gestión en el área administrativa-financiera; el $42 \%$ de los docentes dice que siempre los directivos mantienen liderazgo y gestión en el área administrativa-financiera; y el $8 \%$ de los docentes dice que nunca los directivos mantienen liderazgo y gestión en el área administrativa-financiera.

Figura 8. Los Valores

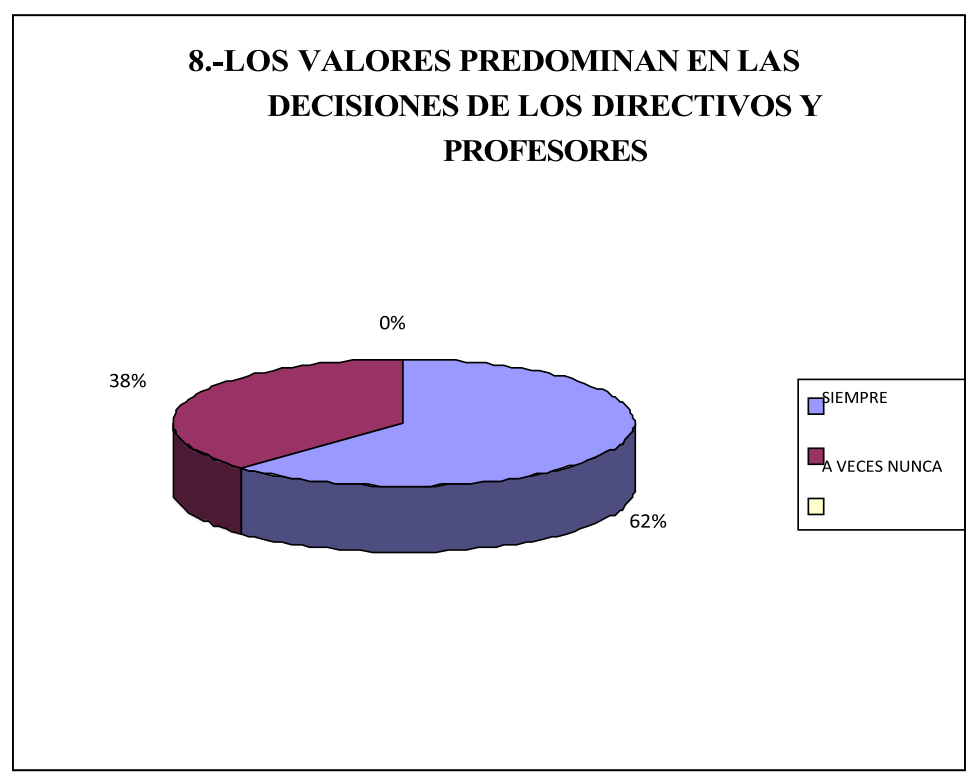

Fuente: Elaboración propia.

En la figura 8 se aprecia que el $62 \%$ de los docentes dice que siempre los valores predominan en las decisiones de los directivos y profesores; el $38 \%$ de los docentes dice que a veces los valores predominan en las decisiones de los directivos y profesores; mientras que el $0 \%$ dice que nunca los valores predominan en las decisiones de los directivos y profesores.

\section{Discusión.}

Los resultados expuestos permiten discutir acerca de lo situación de la escuela, y por ello de la implementación de la gestión del conocimiento; dentro de este contexto los equipos de trabajo en la escuela están dirigidos por los coordinadores de área, en el caso de la Escuela de Física y Matemática hay las áreas básica y las de ejercicio profesional, esto ayuda a trabajar en conjunto con todos.

Las tareas de los miembros de la escuela se encuentran escritas en el manual de normas, reglas y procedimientos, es decir en el estatuto politécnico descrito anteriormente.

El clima de respeto y consenso en la toma de decisiones está liderado por el director, quien 
es el líder y toma un papel muy importante para poder desarrollar todas las actividades establecidas.

Para la resolución de conflictos y promover soluciones pertinentes y oportunas en el tiempo, el director delega la toma de decisiones a un grupo de colaboradores y este grupo está integrado por docentes y estudiantes lo que significa que si hay una organización dentro de la escuela.

Siempre se promueve la excelencia académica, el desarrollo profesional de los docentes, a veces se trabaja mejor en equipo, y siempre hay delegación de funciones, como en algunas instituciones algunas personas nos sentimos bien a veces trabajando en equipo y otras veces proferimos hacerlo solos pero siempre pensando en la excelencia académica.

Las habilidades para dirigir son innatas aunque también la experiencia y el conocimiento adquirido son muy importantes, como habíamos dicho el líder es una persona que actúa como guía o jefe de grupo. Para que su liderazgo sea efectivo, el resto de los integrantes debe reconocer sus capacidades, y estas son mejoradas con la experiencia propia y de otros.

Para mejorar el desempeño y el progreso de la escuela de Física y Matemática el director promueve siempre el uso de la información de resultados de desempeño docente, estudiantes y directivos como referencia para saber qué les falta mejorar, y también la existencia de ambientes cordiales de trabajo.

El equipo educativo o equipo didáctico o junta de profesores de la escuela es el encargado de siempre llevar a cabo la evaluación o seguimiento global del grupo de alumnos, establecer las acciones necesarias para mejorar el clima de convivencia del grupo y coordinar las actividades de enseñanza y aprendizaje que se proponga a los alumnos; este equipo también a veces se encarga de tratar de forma coordinada los conflictos que puedan surgir en el grupo y establecer las medidas oportunas para resolverlos.

La gestión pedagógica en la escuela de física y matemática, fomenta la producción de diagnósticos y de soluciones propias y adecuadas a la diversidad y potenciales de la comunidad y del entorno geográfico.

En cuanto a la investigación realizada sobre los docentes podemos discutir; la mayoría de los docentes pensamos que a veces el rol del docente líder se define como una persona que posee la actitud y las habilidades para cuestionar las órdenes ya existentes, es verdad que un líder posee ciertas habilidades pero no sólo en el plano de discutir si no también para opinar y proponer actividades para alcanzar metas.

En su mayoría la gerencia educativa se promueve a todo nivel dentro de lo educativo 
brindando un ambiente de aprendizaje agradable, armónico, seguro y estimulante, el papel del gerente en este caso del director de escuela es muy importante para crear buenos ambientes.

La mayoría piensa que el trabajo en equipo ayuda a tomar decisiones en el cambio de metodologías en el proceso de enseñanza-aprendizaje, trabajar en equipo es importante pues siempre es importante tomar en cuenta la opinión de los involucrados dentro del cambio.

\section{Conclusiones.}

- A través de las encuestas se identificó y se organizó el conocimiento existente

- Se ha logrado facilitar la creación de nuevo conocimiento fundamentado en el liderazgo y los valores.

- Se ha implementado la gestión del conocimiento con liderazgo y valores dentro de la Escuela de Física y Matemática de la ESPOCH.

\section{Referencias bibliográficas.}

Bennis, W. y Burt N. (1985). Líderes: el arte de mandar. Merlin Libros. México.

Brugges, W. (1988). Diccionario de filosofía. Herder, Barcelona.

Buele Maldonado, M. (2011). Instructivo para la elaboración de tesis, proyecto de grado II, Loja Ecuador.

Camargo, M. y Dussán, M. (2002). Investigación Educativa e Innovación Pedagógica.

Colombia Ciencia y Tecnología - COLCIENCIA. Bogotá.

Chavarría Olarte, M. (2007). Educación en un mundo globalizado. Editorial Trillas, México.

D’ Angelo, O. (1996). Provida. Autorrealización de la personalidad. Ed. Academia. La Habana.

Diccionario de la Lengua Española. (2001). Larousse, México.

Fipella, Y. y Ramón Pes Puig. (1987). Liderazgo Transformacional. Revista "Alta Dirección", No. 133.

Frigerio. G, Poggi. M, y otras. (1992). Las instituciones educativas, Cara y ceca. Ed. Troquel. Bs.As. Argentina.

González, J. (1988). El Liderazgo, un punto de vista para su estudio", CETDIR, La Habana. Harris y Hopkins. (2002). Modelo de gestión educativa. México.

Hartman, Robert S. (1997). La estructura del valor, FCE, México.

John, K. (1996). El liderazgo como proceso de transformación dentro de las organizaciones.

Latapi, P. (2000). Valores y educación, UNAM. México. 
López de Llergo, A. (2000). Valores, valoraciones y virtudes, CECSA, México. MOORE, T. W. (1996). Educación en valores. Madrid. Grupo Anaya Editorial. Primera edición.

Sayles, L. (1992). Liderazgo.Mc Graw-Hill, México.

\section{PARA CITAR EL ARTÍCULO INDEXADO.}

Altamirano Novillo, T., Valverde Aguirre, P., Sánchez Vimos, J. E., \& Aguilar Reyes, J. (2019). Implementación de la gestión del conocimiento con liderazgo y valores en una IES del Ecuador. Ciencia Digital, 3(3.2.1), 31-42. https://doi.org/10.33262/cienciadigital.v3i3.2.1.780

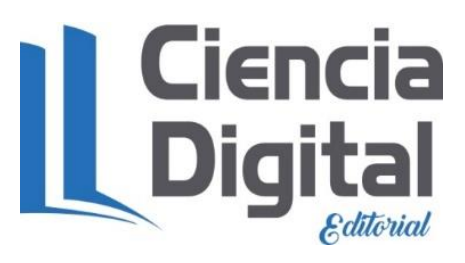

El artículo que se publica es de exclusiva responsabilidad de los autores y no necesariamente reflejan el pensamiento de la Revista Ciencia Digital.

El artículo queda en propiedad de la revista y, por tanto, su publicación parcial y/o total en otro medio tiene que ser autorizado por el director de la Revista Ciencia Digital.
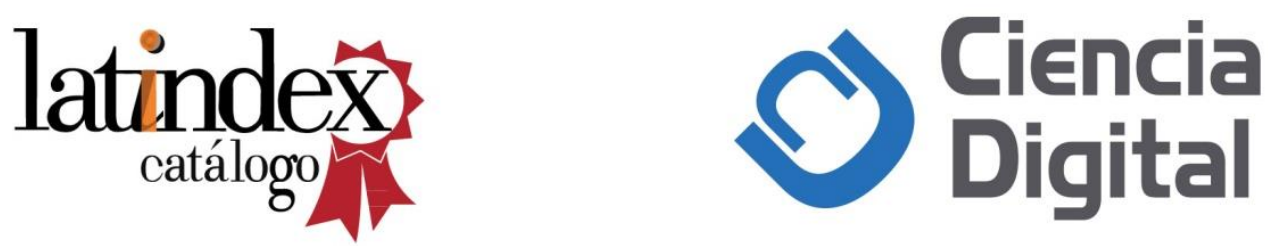